\title{
Advances in Therapeutic Electroporation to Mitigate Muscle Contractions
}

\author{
Christopher B. Arena and Rafael V. Davalos*
}

Bioelectromechanical Systems Lab, Virginia Tech-Wake Forest School of Biomedical Engineering and Sciences, Virginia Tech, 329 ICTAS Building, (MC0298), Stranger Street, Blacksburg, VA 24061, USA

\begin{abstract}
There is a growing effort among researchers to develop unique ways to apply pulsed electric fields for therapeutic applications that reduce the intensity or extent of muscle contractions in order to eliminate the use of muscle relaxants in clinical practice.
\end{abstract}

Keywords: Irreversible Electroporation; Focal ablation; Reversible electroporation; Electro chemotherapy; Pulsed Electric Field; Bipolar pulses; Muscle contractions; Nerve stimulation

Abbreviations: PEF: Pulsed Electric Field; nsPEF: nanosecond Pulsed Electric Field; IRE: Irreversible Electroporation; EBT: Electroporation-Based Therapy; ECT: Electrochemotherapy

\section{Introduction}

Electroporation is a phenomenon resulting from the application of an electric field across cell membranes, where in structural defects, commonly referred to as nanopores [1], are formed in response to an elevated transmembrane potential. The external electric field is conventionally unipolar, and its parameters can be regulated to create reversible defects, which enhance the permeability of the plasma membrane to molecules without compromising viability, or irreversible defects that ultimately lead to cell death. Currently, both modalities are being implemented as a therapeutic means to combat cancer. In applications of reversible electroporation, favorable clinical results have been obtained when low dosages of chemotherapeutic agents [2] or plasmid DNA [3] are used in combination with pulsed electric fields (PEFs). Alternatively, irreversible electroporation (IRE), performed with a comparatively higher field strength, duration, or pulse number, has been recognized as a non-thermal tissue ablation modality [4] capable of treating clinical tumors without adjuvant molecules [5].

Electroporation-based therapies (EBTs) are gaining interest as viable alternatives to surgical resection, chemotherapy, radiation therapy and thermal ablation techniques such as radiofrequency ablation, cryoablation or high-intensity focused ultrasound. As opposed to highly-toxic chemotherapy and radiation therapy regimes, all EBTs are well-tolerated by patients due to a lack of post-procedural complications $[5,6]$. Because the mechanism of cell death does not rely on thermal processes, outcomes are not subject to heat sink effects from blood perfusion, which can protect tumors from thermal therapies. Additionally, the treatment volume is predictable based on the electric field distribution in the tissue $[7,8]$. Specific to IRE, there is a distinct demarcation between ablated and non-ablated tissue that is visible in real-time on multiple imaging platforms $[9,10]$. Within the IRE ablation zone, it has been shown that extracellular matrix components are spared when parameters are chosen to avoid thermal damage. This permits treatment of surgically inoperable tumors in close proximity to major blood vessels and nerves $[9,11,12]$ and the rapid repopulation of healthy cells post-IRE [10].

A challenge with EBTs is that the delivery of unipolar electric pulses characteristic of electroporation causes muscle contractions. To reduce movement, muscle relaxants may be administered to patients prior to treatment $[10,13-15]$. This poses additional concerns for the anesthesiologist, as the dosage of muscle relaxants must be continually monitored to ensure an adequate neuromuscular blockade and proper respiratory function [16]. Electrically induced movement, while not ideal, has not prevented EBTs from being established as safe and effective treatment options for cancer $[2,3,5,17,18]$ and other non-cancerous pathologies [19]. However, there has been a growing emphasis in the literature on developing novel techniques for performing electroporation that reduce the intensity or extent of muscle contractions [20-24].

\section{Current State of the Art}

Perhaps the first attempt to reduce muscle contractions during EBTs was made by Daskalov et al. [21]. Stemming from their work in the field of electrical stimulation [25], the authors designed a bipolar pulse generator to perform reversible electroporation with chemotherapeutic agents, or electrochemotherapy (ECT). The bipolar pulses consisted of a rectangular positive phase (50 $\mu$ s duration) followed immediately by a negative phase of equivalent duration. The system was tested at electric field strengths up to $1250 \mathrm{~V} / \mathrm{cm}$ on patients with basal cell and spin cell carcinoma by delivering eight individual pulses at $1 \mathrm{~s}$ intervals or a single burst of eight pulses with $1 \mathrm{~ms}$ spacing. For comparison to conventional electroporation protocols, eight unipolar rectangular pulses ( $100 \mu$ s duration) were also delivered at 1 $s$ intervals in some tumor locations. Only a local anesthetic was used in the procedure without any muscle relaxant. All patients responded positively and completely to treatment, regardless of the implemented pulsing protocol. While the authors make no comment on the severity or extent of muscle contractions, they do note that bipolar pulses were better tolerated by patients. Additionally, for the application of eight pulses as a single burst, the patients experienced only a single electrical sensation, as opposed to series of eight [21]. The technique of raising the pulse repetition rate above the frequency of tetanic contraction was later confirmed to be an effective option for unipolar pulsing as well $[18,26]$, assuming optimal drug dosing [27].

*Corresponding author: Rafael V. Davalos, Bioelectromechanical Systems Lab, Virginia Tech-Wake Forest School of Biomedical Engineering and Sciences, Virginia Tech, 329 ICTAS Building, (MC0298), Stranger Street, Blacksburg, VA 24061, USA, Tel: +1-540-231-1979; Fax: +1-540-541-8320; E-mail: davalos@vt.edu

Received March 13, 2012; Accepted March 15, 2012; Published March 20, 2012

Citation: Arena CB, Davalos RV (2012) Advances in Therapeutic Electroporation to Mitigate Muscle Contractions. J Membra Sci Technol 2:e102. doi:10.4172/2155 9589.1000e102

Copyright: (C) 2012 Arena CB, et al. This is an open-access article distributed under the terms of the Creative Commons Attribution License, which permits unrestricted use, distribution, and reproduction in any medium, provided the original author and source are credited. 
While applying bursts of microsecond-long unipolar pulses can reduce the total number of muscle contractions per treatment, the force of contraction is similar to that generated during an individual unipolar pulse [26]. However, as alluded to by Daskalov et al. [21], altering pulse polarity can be employed to reduce the intensity of muscle contractions. According to classic literature on electrical stimulation, a bipolar pulse has a higher current threshold for action potential excitation as compared to a unipolar pulse of equivalent phase duration [28]. This effect is enhanced as pulse duration is reduced. When a microsecond order pulse is applied, there is a latency period between the offset of the pulse and the rising phase of the action potential. A rapid reversal of polarity falling within this latency period can accelerate passive repolarization and inhibit action potential generation [29]. Therefore, with proper tuning of bipolar pulse parameters, it is possible to achieve a drop in muscle force, which can be attributed to the cessation of action potentials in part of the motor unit population.

Our group invented a technique that utilizes bipolar pulses with a phase duration an order of magnitude shorter than that used by Daskalov et al. [21], to induce IRE without causing muscle contractions [20]. In this study, a pulse generator was developed that could deliver rectangular pulses with phase durations as short as $1 \mu \mathrm{s}$. It is important to note that multiple cycles of bipolar pulses were applied in succession to generate a $200 \mu$ s burst that was repeated once per second. The pulses were applied through electrodes inserted into the sensorimotor cortex of rats in order to study both direct stimulation of surrounding neck musculature through leak currents and induction of limb movement through stimulation of efferent neuronal pathways [12]. Qualitative assessment of the treated regions viewed histologically and under MRI indicated that bipolar pulses could non-thermally ablate tissue, albeit at a higher electric field strength when compared to unipolar pulses. However, even at higher field strengths (up to $4000 \mathrm{~V} / \mathrm{cm}$ ), there was no visual or tactile evidence of muscle contractions when bipolar pulses with phase durations of 1 to $2 \mu$ s were tested. Conversely, all IRE protocols tested with $200 \mu$ s long unipolar pulses produced detectable muscle contractions at field strengths as low as $500 \mathrm{~V} / \mathrm{cm}[20]$. In future work, additional experiments should be performed over the skin and in muscle for direct comparison to $[21,29]$.

When pulse duration is reduced even further into the nanosecond range and stronger electric fields $(10-100 \mathrm{kV} / \mathrm{cm})$ are applied, it becomes possible to permeabilize both the plasma membrane intracellular structures [30]. These nanosecond pulsed electric fields (nsPEFs)have also shown great promise for treating cancer [31,32] by promoting calcium bursts [33], translocation of phosphatidylserine [33], and DNA damage [34], all of which are capable of triggering an apoptotic cascade. Typically, the pulse generator is based on a spark gap switched transmission line, and the resulting output closely resembles a unipolar pulse $[35,36]$. For unipolar pulses of this nature, cell death can be induced with a significant drop in muscle contractions as compared to unipolar microsecond-long pulses[23]. Experimentally derived strength-duration curves indicate that a $100 \mathrm{~ns}$ pulse requires a voltage two orders of magnitude greater than a $10 \mu$ s pulse to excite muscle [37].

Nerve conduction block induced by electrical currents, as opposed to chemical muscle relaxants, is another, largely unexplored, option for mitigating muscle contractions during EBTs. While the biophysics are not completely understood, scientists have shown that a reversible, fast-acting peripheral nerve block can be obtained with high-frequency bipolar pulses $[38,39]$ or unipolar nsPEFs [40] without any accompanying thermal damage. It is thought that disrupting the transmembrane potential across a nerve at a localized point downstream from excitation may inhibit action potential propagation. Practical application of electrically mediated nerve blocking would require the placement of additional electrodes adjacent to those used for EBTs. If performed correctly, this technique could offer a safer alternative to muscle relaxant administration.

Recently, an alternative technique for mitigating muscle contractions during IRE has been proposed by Goldberg and Rubinsky [22]. Utilizing the concept of a Faraday cage, the authors have found that a certain arrangement of needle electrodes limits the amount of tissue exposed to electric fields above the threshold for muscle contraction, while having a minimal impact on the extent of electroporation. The design consists of a central energized electrode surrounded by an array of grounded electrodes. Similar geometries have been used successfully for cardiac defibrillation [41] and ECT [42]. Interestingly, by having 16 or more grounded electrodes and by reducing the insertion depth of the central energized electrode relative to the grounded electrodes, the predicted amount of tissue experiencing muscle contractions falls dramatically [22]. Upon in vivo translation, it is expected that this concept will reduce direct stimulation of denervated muscle, as suggested here, and also reduce motor unit recruitment from nerve stimulation outside the targeted electroporated zone.

\section{Conclusion}

The reports presented above indicate that many feasible routes exist for mitigating muscle contractions during EBTs. Continued development of all of these routes will be beneficial, as there may be certain applications for which some options are better suited. For example, the electrode configuration proposed above [22] requires an increase in the number of electrodes and may be most valuable in situations where the targeted region can be treated non-invasively, such as applications over the skin. Additionally, while positive results have been obtained with bipolar pulsing protocols in terms of reducing or eliminating muscle contractions, more work needs to be done in optimizing pulse parameters for specific applications. In a more recent study on ECT with bipolar pulses [43], a reduction in phase duration below $50 \mu$ reduced electrical sensation, but also had a negative impact on treatment efficacy. However, we have shown that IRE can be performed with a phase duration of $1 \mu$ s by increasing the number of pulses and electric field strength [20]. Along these same lines, it should also be theoretically possible to cause cell death with bipolar nsPEFs of sufficient field strength. This may present an added improvement over the reduction in muscle contractions already seen with unipolar nsPEFs [23]. In the future, it is likely that continued work on mitigating muscle contractions will lead to the commercialization of new pulse generators and electrode designs that eliminateany need for muscle relaxant administration. This will further facilitate the widespread use of EBTs in clinical practice.

\section{References}

1. Weaver JC, Chizmadzhev YA (1996) Theory of electroporation: A review. Bioelectrochem Bioenerg 41: 135-160.

2. Mir LM, Belehradek M, Domenge C, Orlowski S, Poddevin B, et al. (1991) Electrochemotherapy, a new antitumor treatment: first clinical trial. C R Acad Sci III 313: 613-618.

3. Daud Al, DeConti RC, Andrews S, Urbas P, Riker Al, et al. (2008) Phase Trial of Interleukin-12 Plasmid Electroporation in Patients With Metastatic Melanoma. J Clin Oncol 26: 5896-5903.

4. Davalos RV, Mir LM, Rubinsky B (2005) Tissue ablation with irreversible electroporation. Ann Biomed Eng 33: 223-231.

5. Thomson KR, Cheung W, Ellis SJ, Federman D, Kavnoudias H, et al. (2011) Investigation of the Safety of Irreversible Electroporation in Humans. J Vasc Interv Radiol 22: 611-621. 
Citation: Arena CB, Davalos RV (2012) Advances in Therapeutic Electroporation to Mitigate Muscle Contractions. J Membra Sci Technol 2:e102. doi:10.4172/2155-9589.1000e102

6. Spugnini EP, Vincenzi B, Citro G, Dotsinsky I, Mudrov T, et al. (2011) Evaluation of Cisplatin as an Electrochemotherapy Agent for the Treatment of Incompletely Excised Mast Cell Tumors in Dogs. J Vet Intern Med 25: 407-411.

7. Miklavcic D, Snoj M, Zupanic A, Kos B, Cemazar M, et al. (2010) Towards treatment planning and treatment of deep-seated solid tumors by electrochemotherapy. Biomed Eng Online 9: 10

8. Edd JF, Davalos RV (2007) Mathematical Modeling of irreversible Electroporation for treatment planning. Technology in Cancer Research \& Treatment 6: 275-286.

9. Garcia PA, Pancotto T, Rossmeisl JH, Henao-Guerrero N, Gustafson NR, et al. (2011) Non-Thermal Irreversible Electroporation (N-TIRE) and Adjuvant Fractionated Radiotherapeutic Multimodal Therapy for Intracranial Malignant Glioma in a Canine Patient. Technology in Cancer Research \& Treatment 10 73-83.

10. Rubinsky B, Onik G, Mikus P (2007) Irreversible Electroporation: A New Ablation Modality - Clinical Implications. Technol Cancer Res Treat 6: 37-48.

11. Neal RE II, Rossmeis JH, Garcia PA, Lanz OI, Henao-Guerrero N, et al (2011) Successful Treatment of a Large Soft Tissue Sarcoma With Irreversible Electroporation. J Clin Oncol 29: e372-377.

12. Maor E, Ivorra A, Leor J, Rubinsky B (2007) The effect of irreversible electroporation on blood vessels. Technol Cancer Res Treat 6: 307-312.

13. Ball C, Thomson KR, Kavnoudias H (2010) Irreversible Electroporation: A New Challenge in "Out of Operating Theater" Anesthesia. Anesthesia and Analgesia 110: $1305-1309$

14. Landstrom FJ, Nilsson COS, Crafoord S, Reizenstein JA, Adamsson GBM, et al. (2010) Electroporation Therapy of Skin Cancer in the Head and Neck Area. Dermatologic Surgery 36: 1245-1250.

15. Billard V, Garbay JR, Morsli N, Robert C, Mir L, et al. (2004) Sedation and anesthesia for electrochemotherapy. Eurocancer John Libbey Eurotext Paris: 229-230.

16. Eikermann M, Groeben H, Husing J, Peters J (2003) Accelerometry of adductor pollicis muscle predicts recovery of respiratory function from neuromuscular blockade. Anesthesiology 98: 1333-1337.

17. Ferraro B, Morrow MP, Hutnick NA, Shin TH, Lucke CE, et al. (2011) Clinical Applications of DNA Vaccines: Current Progress. Clinical Infectious Diseases 53: 296-302.

18. Marty M, Sersa G, Garbay JR, Gehl J, Collins CG, et al. (2006) Electrochemotherapy - An easy, highly effective and safe treatment of cutaneous and subcutaneous metastases: Results of ESOPE (European Standard Operating Procedures of Electrochemotherapy) study. Ejc Supplements 4: 3-13.

19. Maor E, Ivorra A, Rubinsky B (2009) Non Thermal Irreversible Electroporation: Novel Technology for Vascular Smooth Muscle Cells Ablation. "PloS One" 4: e4757.

20. Arena CB, Sano MB, Rossmeisl JH, Caldwell JL, Garcia PA, et al. (2011) High-Frequency Irreversible Electroporation (H-FIRE) for Non-thermal Ablation without Muscle Contraction. Biomed Eng Online 10: 102.

21. Daskalov I, Mudrov N, Peycheva E (1999) Exploring new instrumentation parameters for electrochemotherapy - Attacking tumors with bursts of biphasic pulses instead of single pulses. IEEE Eng Med Biol Mag 18: 62-66.

22. Golberg A, Rubinsky B (2012) Towards electroporation based treatment planning considering electric field induced muscle contractions. Technol Cancer Res Treat 11: 189-201.

23. Long G, Shires PK, Plescia D, Beebe SJ, Kolb JF, et al. (2011) Targeted Tissue Ablation With Nanosecond Pulses. IEEE Transactions on Biomed Eng 58.

24. Nikolova B, Tsoneva I, Peycheva E (2011) Treatment of Melanoma by Electroporation of Bacillus Calmette-Guerin. Biotechnol \& Biotechnol Equip 25: 2522-2524.

25. Daskalov I, Bankov S (1997) Electrical Stimulation of Innervated Muscles. J Clin Eng 22: 383-390.

26. Miklavcic D, Pucihar G, Pavlovec M, Ribaric S, Mali M, et al. (2005) The effect of high frequency electric pulses on muscle contractions and antitumor efficiency in vivo for a potential use in clinical electrochemotherapy. Bioelectrochemistry 65: $121-128$

27. Sersa G, Kranjc S, Scancar J, Krzan M, Cemazar M (2010) Electrochemotherapy of Mouse Sarcoma Tumors Using Electric Pulse Trains with Repetition Frequencies of $1 \mathrm{~Hz}$ and $5 \mathrm{kHz}$. J Membr Biol 236: 155-162.

28. Reilly JP, Freeman VT, Larkin WD (1985) Sensory Effects of Transien Electrical-Stimulation - Evaluation with a Neuroelectric Model. IEEE Trans Biomed Eng 32: 1001-1011.

29. van den Honert C, Mortimer JT (1979) Response of the Myelinated Nerve-Fibe to Short Duration Biphasic Stimulating Currents. Ann Biomed Eng 7: 117-125.

30. Beebe SJ, Fox PM, Rec LJ, Willis LK, Schoenbach KH (2003) Nanosecond high-intensity pulsed electric fields induce apoptosis in human cells. FASEB 17: $1493-1495$

31. Garon EB, Sawcer D, Vernier PT, Tang T, Sun YH, et al. (2007) In vitro and in vivo evaluation and a case report of intense nanosecond pulsed electric field as a local therapy for human malignancies. Int J Cancer 121: 675-682.

32. Nuccitelli R, Pliquett U, Chen XH, Ford W, Swanson RJ, et al. (2006) Nanosecond pulsed electric fields cause melanomas to self-destruct. Biochem Biophys Res Commun 343: 351-360.

33. Vernier PT, Sun YH, Marcu L, Salemi S, Craft CM, et al. (2003) Calcium bursts induced by nanosecond electric pulses. Biochem Biophys Res Commun 310 286-295.

34. Stacey M, Stickley J, Fox P, Statler V, Schoenbach K, et al. (2003) Differentia effects in cells exposed to ultra-short, high intensity electric fields: cell survival, DNA damage, and cell cycle analysis. Mutation Research-Genetic Toxicology and Environmental Mutagenesis 542: 65-75.

35. Behrend M, Kuthi A, Gu XY, Vernier PT, Marcu L, et al. (2003) Pulse generators for pulsed electric field exposure of biological cells and tissues. IEE Trans Dielectr and Electrical Insulation 10: 820-825.

36. Kolb JF, Kono S, Schoenbach KH (2006) Nanosecond pulsed electric field generators for the study of subcellular effects. Bioelectromagnetics 27: 172 187.

37. Rogers WR, Merritt JH, Comeaux JA, Kuhnel CT, Moreland DF, et al. (2004) Strength-duration curve for an electrically excitable tissue extended down to near 1 nanosecond. leee Transactions on Plasma Science 32: 1587-1599.

38. Kilgore KL, Bhadra N (2004) Nerve conduction block utilising high-frequency alternating current. Medical \& Biological Engineering \& Computing 42: 394-406.

39. Tai CF, de Groat WC, Roppolo JR (2005) Simulation analysis of conduction block in unmyelinated axons induced by high-frequency biphasic electrical currents. IEEE Trans Biomed Eng 52: 1323-1332.

40. Joshi RP, Mishra A, Song J, Pakhomov AG, Schoenbach KH (2008) Simulation studies of ultrashort, high-intensity electric pulse induced action potential block in whole-animal nerves. IEEE Trans Biomed Eng 55: 1391-1398.

41. Jayanti V, Zviman MM, Nazarian S, Halperin HR, Berger RD (2007) Nove electrode design for potentially painless internal defibrillation also allows for successful external defibrillation. Journal of Cardiovascular Electrophysiology 18: 1095-1100.

42. Sersa G, Miklavcic D, Cemazar M, Rudolf Z, Pucihar G, et al. (2008) Electrochemotherapy in treatment of tumours. Ejso 34: 232-240.

43. Peycheva E, Daskalov I (2004) Electrochemotherapy of skin tumours: comparison of two electroporation protocols. J BUON 9: 47-50. 